\title{
视频图像运动估计中的一维块匹配算法
}

\author{
刘泉洋, 刘云清*, 史俊, 颜飞, 张琼 \\ (长春理工大学电子与信息工程学院 长春 130022) \\ (mzlyq@cust.edu.cn)
}

\begin{abstract}
摘 要: 运动估计是视频图像压缩和视频图像修复等领域的基础问题, 传统的块匹配法搜索质量较好, 但搜索速度 不够快. 针对传统块匹配法搜索速度上的不足, 提出一种快速的一维块匹配运动估计算法. 首先对运动矢量正交分 解, 使用特殊权重系数矩阵对二维匹配块做降维处理, 得到 2 组一维特征矩阵; 然后选择一维三步搜索法作为搜索策 略, 最小绝对误差和准则作为匹配准则, 使用 2 组一维特征矩阵搜索匹配运动矢量的 2 个分量; 最后将分量组成完整 的运动矢量. 通过多组对比实验的结果表明, 该算法在保证定量评价 PSNR 的前提下, 显著提升运动估计的搜索速 度, 视频清晰度越高、匹配块像素尺寸越大, 运动估计搜索速度提升越明显.
\end{abstract}

关键词：运动估计; 块匹配算法; 正交分解; 特征矩阵; 三步搜索法

中图法分类号: TP391.41 DOI: $10.3724 /$ SP.J.1089.2021.18343

\section{One-dimensional Block Matching Algorithm in Video Image Motion Estimation}

\author{
Liu Quanyang, Liu Yunqing*, Shi Jun, Yan Fei, and Zhang Qiong \\ (School of Electronics and Information Engineering, Changchun University of Science and Technology, Changchun 130022)
}

\begin{abstract}
Motion estimation is a basic problem in the fields of video image compression and video image restoration. The traditional block matching methods have good search quality, but the search speed is not fast enough. Aiming at the shortcomings of the search speed in the traditional block matching methods, we proposed a fast one-dimensional block matching motion estimation algorithm. Firstly, the motion vector is orthogonally decomposed, and the two-dimensional matching block is reduced by a special weight coefficient matrix to obtain two sets of one-dimensional feature matrices. Then the one-dimensional three step search method is selected as the search strategy. The sum of absolute differences criterion is used as the matching criterion. The two sets of one-dimensional feature matrices are used to search for the two components of the matching motion vector. Finally the two components are formed into a complete motion vector. The results of multiple sets of comparative experiments show that the search speed of motion estimation is significantly improved while the algorithm is guaranteed to quantitatively evaluate the PSNR. The higher the video definition and the larger the pixel size of the matching block, the better the algorithm can improve the search speed of motion estimation.
\end{abstract}

Key words: motion estimation; block matching algorithm; orthogonal decomposition; feature matrix; three-step search

收稿日期：2020-05-04; 修回日期：2021-01-08. 基金项目：吉林省科技厅重点项目(20190303080SF，20190303034SF). 刘泉洋 (1995一), 男, 硕士研究生, 主要研究方向为传感与信号处理; 刘云清(1970一), 男, 博士, 博士生导师, 论文通讯作者, 主要研究方 向为智能信息处理、自动控制; 史俊(1996一), 男, 硕士研究生, 主要研究方向为模式识别与智能系统; 颜飞(1987一), 男, 博士, 硕士 生导师, 主要研究方向为智能信息处理; 张琼(1991一), 女, 博士, 讲师, 主要研究方向为数据处理. 
运动估计是视频修复和视频压缩的关键技术， 其主要目的是利用图像帧间信息修复视频并减少 图像帧间的信息冗余 ${ }^{[1-2]}$. 目前, 已有很多运动估 计算法，其中，由于块匹配算法(block matching algorithm, BMA)在计算处理和硬件实现上较为容 易 ${ }^{[3]}$ ，因此被许多视频压缩编码标准所采用，如 H.261/3/4 ${ }^{[4]}$ 和 MPEG- $2 / 4^{[5]}$.

BMA 计算量较大, 给实时处理带来较大压力. 为了减少运动估计的计算量, 近些年有很多学者 对块匹配法进行改进. 改进方式主要有 2 种: 一种 是改变匹配块的形状和位置, 但依然使用二维匹 配块进行搜索，如局部区域匹配法 ${ }^{[6]}$ 将中间的匹配 块变为 4 个等大小的小方块, 其准确性和实时性比 BMA 略有提高. 还有许多学者针对搜索策略进行 优化并提出许多不同的搜索方式, 其速度较三步 搜索法(three step search, TSS)有一定的提高，如文 献[7]通过运动矢量概率分布分析, 发现了运动矢 量概率分布具有除中心十字偏置特性以外的方向性 特性，提出了一种快速的双十字搜索运动估计算 法, 在保持相当搜索质量的前提下，与菱形搜索算 法和十字菱形搜索算法相比, 其搜索速度均有提 高. 切换的快速运动估计算法 ${ }^{[8]}$ 采用了提前停止和 选择性搜索技术来提高编码速度, 以小菱形作为起 始搜索模式, 然后过渡到六边形模式, 最后使用正 方形搜索模式进行细化; 该算法对于各种运动情况 的视频序列具有强普适性, 速度也有所提高. 还有 从其他角度优化运动估计算法, 如文献[9]以像素块 为单位，利用块内外点的比例判定前景区域，同时 引人马尔可夫聚类方法进行后处理, 有效地提高了 运动对象的定位精度; 通过对目标函数引人权重系 数增强对残差的鲁棒性, 以进一步提高算法的估计 精度. 此外，文献[9]基于像素掩模的 3 层金字塔构 建序列图像, 并将改进的梯度方法引人到优化过程 中, 提高了算法的实时性. 文献[10]基于运动分解 估算的运动估计算法, 利用矩阵分解原理将全局运 动分解成帧间运动和前帧运动, 保证了场景快速变 化条件下运动估计的准确性和时效性.

这些算法均采用为二维匹配块搜索匹配运动 矢量的最优值, 而完整二维匹配块存在大量的信 息冗余, 会增大搜索匹配过程中的计算量, 很难通 过优化搜索策略和匹配块位置大幅度提升算法运 算速度. 文献[11]提出基于边界灰度投影匹配的全 局运动估计算法，将图像边界水平投影和垂直投 影值作为匹配特征，较好地估计了全局运动参数; 但是其特征提取模型和搜索策略存在缺陷, 不能
有效地提高搜索速度. 为了进一步提高算法的速 度, 降低算法实现的复杂度, 本文提出了一维块匹 配运动估计算法(one-dimensional BMA, OBMA).

\section{1 一维特征矩阵和一维 TSS}

\section{1 一维特征矩阵}

通过对运动矢量的特点进行分析, 运动矢量 精度是单位像素, 方向和大小均不确定. 因此, 本 文采用将运动矢量 $\boldsymbol{V}_{\mathrm{M}}$ 分解为水平方向分量 $\boldsymbol{x}$ 和垂 直方向分量 $\boldsymbol{y}$, 如图 1 所示.

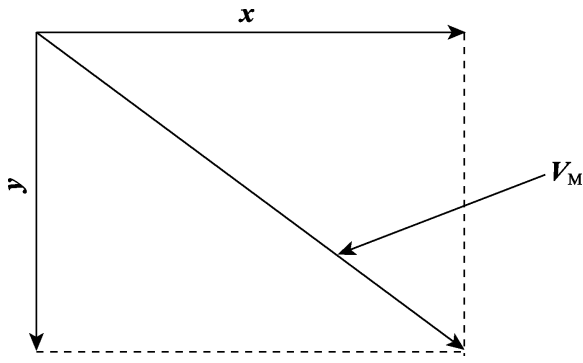

图 1 运动矢量正交分解

求解 $\boldsymbol{x}$ 和 $\boldsymbol{y}$ 需要使用一维特征矩阵 $\boldsymbol{X}$ 和一维 特征矩阵 $\boldsymbol{Y}$, 一维特征矩阵求解过程为

$$
\begin{gathered}
\boldsymbol{A}=[\lambda]_{1 n} \\
\boldsymbol{B}=[\mu]_{1 m} \\
\boldsymbol{X}=\boldsymbol{A P} \\
\boldsymbol{Y}=\boldsymbol{B} \boldsymbol{P}^{\mathrm{T}}
\end{gathered}
$$

其中, $\boldsymbol{A}$ 为权重系数矩阵, $\lambda$ 为 $\boldsymbol{A}$ 的权重系数; $\boldsymbol{B}$ 为权重系数矩阵, $\mu$ 为 $\boldsymbol{B}$ 的权重系数; $\boldsymbol{P}$ 为匹配 块矩阵, 形状为 $(n, m) ; \boldsymbol{X}$ 的形状为 $(1, m) ; \boldsymbol{P}^{\mathrm{T}}$ 形状为 $(m, n) ; \boldsymbol{Y}$ 的形状为 $(1, n)$.

为了更直观地表示一维特征矩阵的特点, 选 取连续 3 帧 $1080 \mathrm{P}$ 测试图像, 从测试图像中提取 $\boldsymbol{P}$. $\lambda$ 和 $\mu$ 设置为 $1 / 256 . \boldsymbol{A}$ 的形状为 $(1,540), \boldsymbol{B}$ 的形状为 $(1,960)$; 得到 3 幅连续测试图像的一维 特征矩阵如图 2 所示. 其中, $\boldsymbol{X}$ 的形状为 $(1,960)$, $\boldsymbol{Y}$ 的形状为 $(1,540)$, 纵坐标表示一维矩阵中每个 元素的数值.

分析图 2 的发现，连续视频图像匹配块的一维 特征矩阵具有整体趋势相似的特点, 利用此特点 进行运动估计，可以有效地减少信息冗余，提高后 续搜索匹配的速度.

\section{2 一维 TSS}

相比于全搜索法(full search, FS)要遍历匹配块 


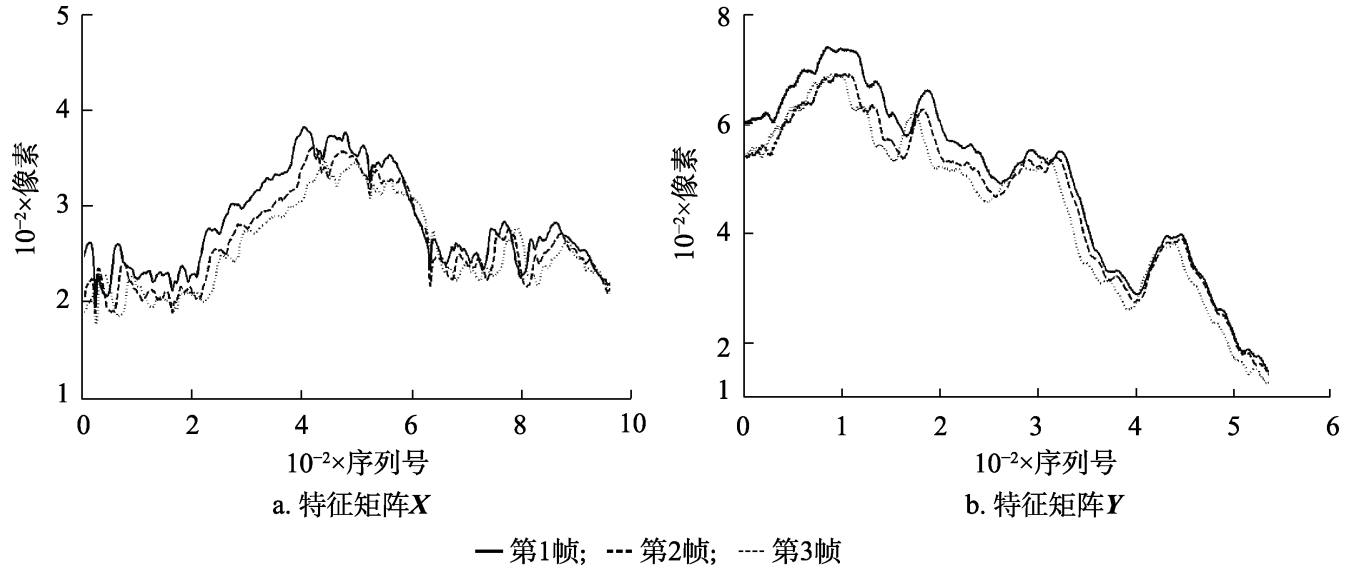

图 2 连续 3 帧视频图像特征矩阵折线图

的所有像素点, TSS 搜索点数大幅减少 ${ }^{[12]}$. 有别于 逐一遍历所有像素点, 如图 3 所示, TSS 每步搜索 对搜索边界上的 8 个点以及正方形的中心点共 9 个搜索点进行比较, 搜索步长等于或者略大于最 大搜索范围的一半; 上一步比较得到的最佳匹配 点作为下一个新的搜索步的搜索中心. 搜索范围 大于 7 时, 搜索步骤不止 3 步.

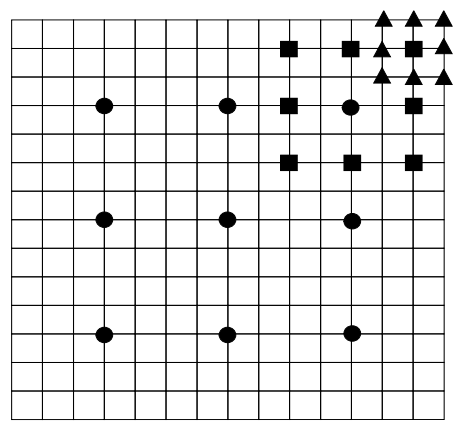

图 3 二维 TSS

本文的特征矩阵 $\boldsymbol{X}$ 和特征矩阵 $\boldsymbol{Y}$ 是一维矩阵, 因此需要将二维 TSS 改为一维 TSS. 一维 TSS 的 搜索步骤与二维 TSS 类似, 每一搜索步对搜索边 界上的 2 个点以及中心点共 3 个搜索点进行比较, 搜索步长等于或者略大于最大搜索范围的一半; 上一步比较后得到的最优匹配点作为下一步的搜 索中心. 一维 TSS 如图 4 所示.

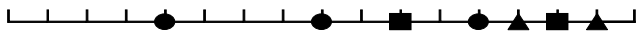

图 4 一维 TSS

一维 TSS 实际使用时需要确定搜索步数, 确 定一维 TSS 搜索步数就是确定搜索半径. 搜索半 径 $r$ 与搜索步数 steps 的关系为

$$
r=2^{\text {steps }}-1
$$

本文算法将 $\boldsymbol{V}_{\mathrm{M}}(x, y)$ 分解为 $\boldsymbol{x}$ 和 $\boldsymbol{y}$, 因此 $\boldsymbol{x}$ 和 $\boldsymbol{y}$ 可以针对不同 $r$ 设置不同的 steps. 测试数据 使用 400 帧清晰度为 $1080 \mathrm{P}$ 的连续视频图像序列, 求出运动矢量, 制作散点图如图 5 所示.

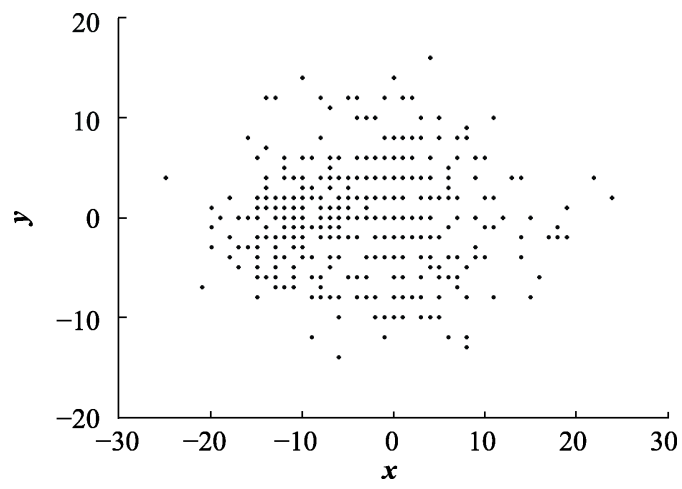

图 $5 \boldsymbol{V}_{\mathrm{M}}$ 散点图

通过图 5 散点图的分析, 散点图中点的整体分 布呈菱形，水平方向的范围大于垂直方向的范围， 在实际的运动估计计算中, 可以针对不同的范围 设置不同的 $r$, 减少不必要的 steps, 提高搜索速 度. 以图 5 为例, 设水平方向的 $r$ 为 $I$, 垂直方向 的 $r$ 为 $J$, 则应设 $I=31, J=15$; 将其分别代人式 (5)求出搜索步数分别为 5 步和 4 步.

\section{OBMA}

\section{1 算法概述}

本文提出的 OBMA 整体流程图如图 6 所示.

\section{2 匹配块}

匹配块选择当前帧图像 $\boldsymbol{S}$ 的中心区域，图像 $\boldsymbol{S}$ 的形状为 $(N, M), \boldsymbol{P}$ 的形状为 $(n, m)$, 在 $\boldsymbol{S}$ 的 位置如图 7 所示. 


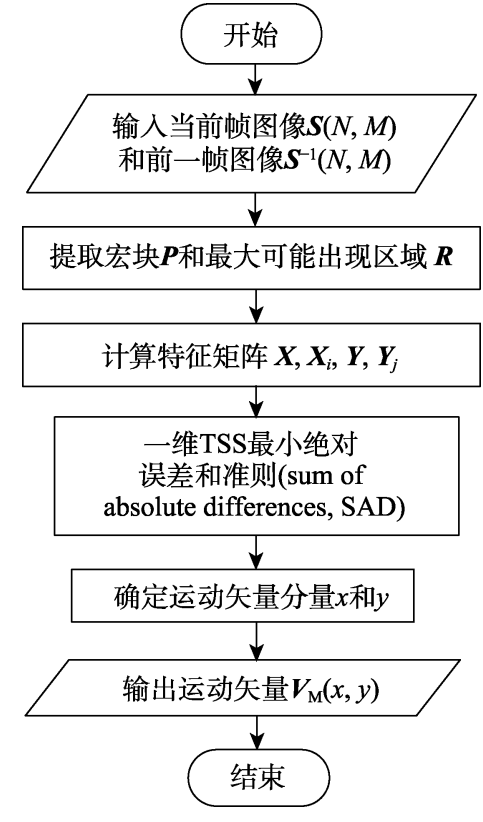

图 6 算法流程图

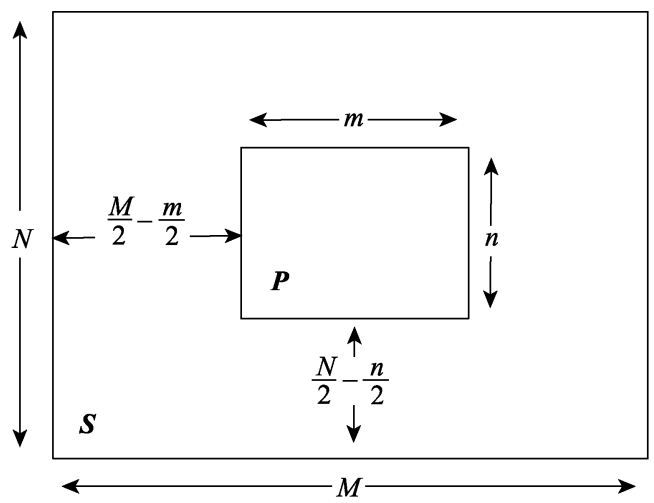

图 7 匹配块

前一帧图像的匹配块记为 $\boldsymbol{P}^{-1}, \boldsymbol{P}^{-1}$ 的最大可 能出现区域用 $\boldsymbol{R}$ 表示，区域 $\boldsymbol{R}$ 包括区域 $\boldsymbol{P}^{-1}$ 以及 水平方向的搜索半径 $I$ 和垂直方向的搜索半径 $J$ 包含的区域, $\boldsymbol{R}$ 的形状为 $(n+2 I, m+2 J)$, 区域 $\boldsymbol{R}$ 如图 8 所示.

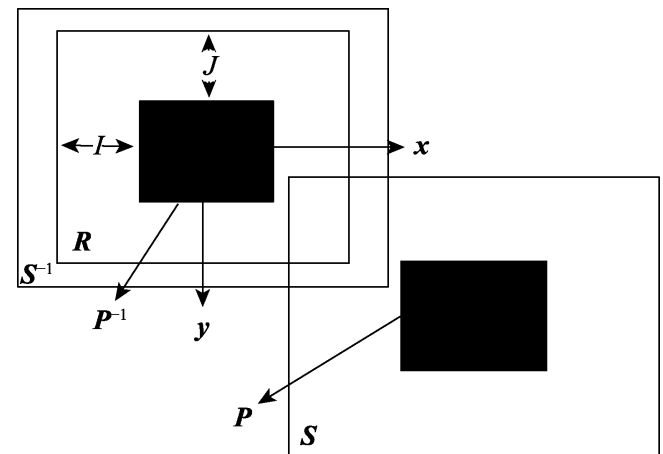

图 8 区域 $\boldsymbol{R}$ 示意图
区域 $\boldsymbol{P}$ 和区域 $\boldsymbol{R}$ 的计算公式分别为

$$
\boldsymbol{P}=\boldsymbol{S}\left(\frac{N}{2}-\frac{n}{2}: \frac{N}{2}+\frac{n}{2}, \frac{M}{2}-\frac{m}{2}: \frac{M}{2}+\frac{m}{2}\right)
$$

$$
\boldsymbol{R}=\boldsymbol{S}^{-1}\left(\frac{N}{2}-\frac{n}{2}-J: \frac{N}{2}+\frac{n}{2}+J, \frac{M}{2}-\frac{m}{2}-I: \frac{M}{2}+\frac{m}{2}+I\right)
$$

其中, $S^{-1}$ 是图像 $S$ 的前一帧图像, $R$ 是图像 $S^{-1}$ 匹配块 $\boldsymbol{P}^{-1}$ 的最大可能出现区域.

\section{3 特征矩阵}

$\boldsymbol{A}$ 和 $\boldsymbol{B}$ 的 $\lambda$ 和 $\mu$ 设为灰度级的倒数. 8 位深度 的图像灰度级为 256, 设 $\lambda=\mu=1 / 256$. 区域 $\boldsymbol{R}$ 的 特征矩阵为

$$
\begin{gathered}
\boldsymbol{X}_{\mathrm{R}}=\left[\frac{1}{256}\right]_{1(n+2 J)} \boldsymbol{R} \\
\boldsymbol{Y}_{\mathrm{R}}=\left[\frac{1}{256}\right]_{1(m+2 I)} \boldsymbol{R}^{\mathrm{T}}
\end{gathered}
$$

其中, $\boldsymbol{X}_{\mathrm{R}}$ 和 $\boldsymbol{Y}_{\mathrm{R}}$ 为区域 $\boldsymbol{R}$ 的特征矩阵; $\boldsymbol{X}_{\mathrm{R}}$ 形状为 $(1, m+2 I), \boldsymbol{Y}_{\mathrm{R}}$ 形状为 $(1, n+2 J)$.

特征矩阵组 $\boldsymbol{X}$ 和 $\boldsymbol{X}_{i}$ 分别为

$$
\begin{gathered}
\boldsymbol{X}=\left[\frac{1}{256}\right]_{1(n)} \boldsymbol{P} \\
\boldsymbol{X}_{i}=\boldsymbol{X}_{\mathrm{R}}(I+i: m+I+i)
\end{gathered}
$$

其中, $\boldsymbol{X}_{i}$ 的形状为 $(1, m) ; i$ 为水平方向的偏移量.

特征矩阵组 $\boldsymbol{Y}$ 和 $\boldsymbol{Y}_{j}$ 分别为

$$
\boldsymbol{Y}=\left[\frac{1}{256}\right]_{1(m)} \boldsymbol{P}^{\mathrm{T}}
$$

$$
\boldsymbol{Y}_{j}=\boldsymbol{Y}_{\mathrm{R}}(J+j: n+J+j)
$$

其中, $\boldsymbol{Y}_{j}$ 的形状为 $(1, n) ; j$ 为垂直方向的偏移量.

\section{4 搜索最优值}

搜索策略使用一维 TSS, 匹配准则使用 $\mathrm{SAD}^{[13]}$. 以运动矢量分量 $\boldsymbol{x}$ 的搜索步数等于 3 为 例，具体搜索步骤如下:

输人. 特征矩阵 $\boldsymbol{X}$ 与 $\boldsymbol{X}_{\boldsymbol{i}}$.

输出. 运动矢量分量 $\boldsymbol{x}$.

Step1. 以 0 中心搜索点, 加上中心点左右步长为 4 的 2 个搜索点, 计算 3 个搜索点 $\boldsymbol{X}$ 与 $\boldsymbol{X}_{i}$ 的 SAD.

Step2. 将上一步的最佳匹配点设为中心搜索点, 计算中心点左右步长为 2 的 2 个搜索点 $\boldsymbol{X}$ 与 $\boldsymbol{X}_{i}$ 的 SAD, 与上一步最佳匹配点比较，更新最佳匹配点.

Step3. 步长改为 1 , 同上一步, 最佳匹配点为 $\boldsymbol{x}$.

运动矢量分量 $\boldsymbol{x}$ 和运动矢量分量 $\boldsymbol{y}$ 除了一 维 TSS 的搜索步数不同, 其他搜索步骤均相同, 这里不再赘述. 


\section{3 实验结果}

为了验证本文提出 OBMA，选择主观评价与 客观指标相结合的评价方式. 主观评价为不同算 法运动补偿后的前后帧差值图像; 客观评价指标 选择峰值信噪比(peak signal to noise ratio, PSNR) 和搜索时间. PSNR 将未加人运动补偿的前后帧差 值图像作为原图像, 加入运动补偿后的前后帧差 值图像为处理后图像.

\section{1 实验平台}

本文进行实验的计算机配置为 AMD Ryzen5
$2600 \mathrm{CPU}(3.40 \mathrm{GHz})$, 内存为 $16 \mathrm{~GB}$; 操作系统为 Windows 10; 编程环境为 Python 3.6.

3.2 主观评价

为了直观地对比本文的 OBMA 与 BMA 的实 际效果, 选取测试视频图像序列中 5 个不同场景, 将未加人运动补偿的前后帧差值图像与加入运动 补偿后的前后帧差值图像进行对比. 考虑差值图 像对比度较低, 为了提高差值图像的对比度, 对样 本的结果进行直方图均衡化处理, 最后得到对比 度增强后的差值图像如图 9 所示. 图 9a 所示为与 前一帧参考帧的差值图像; 图 9b 所示为加人

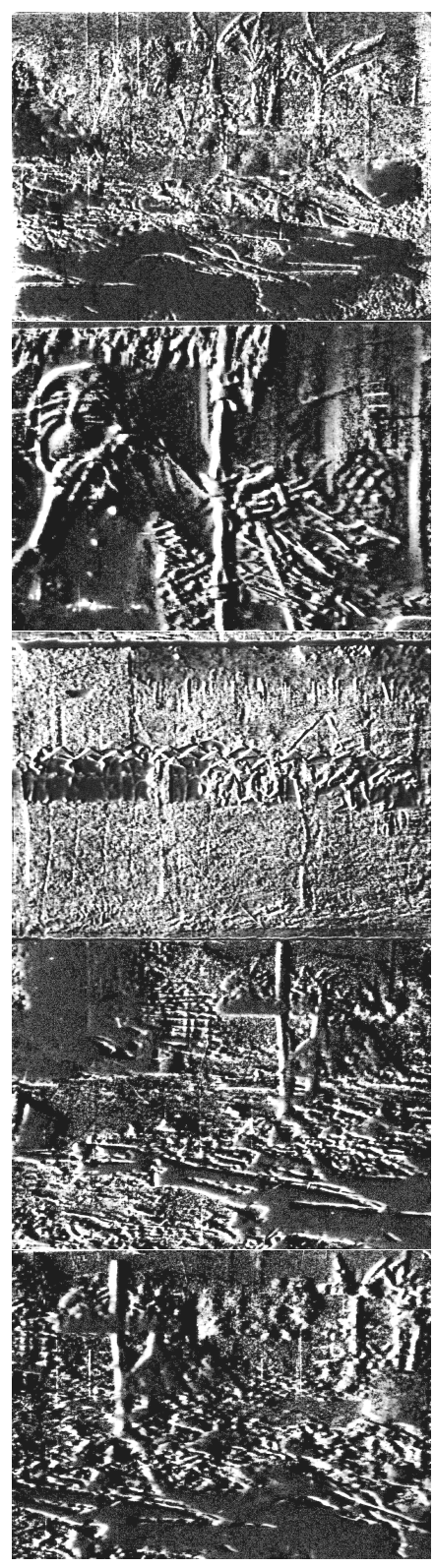

a. 无运动补偿

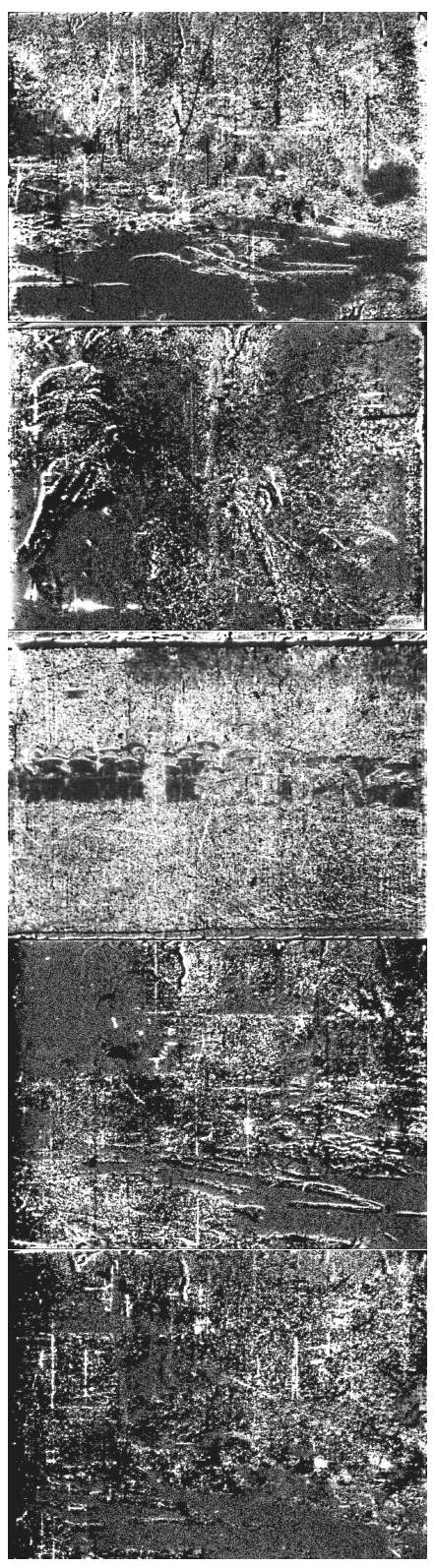

b. $\mathrm{BMA}^{[3]}$

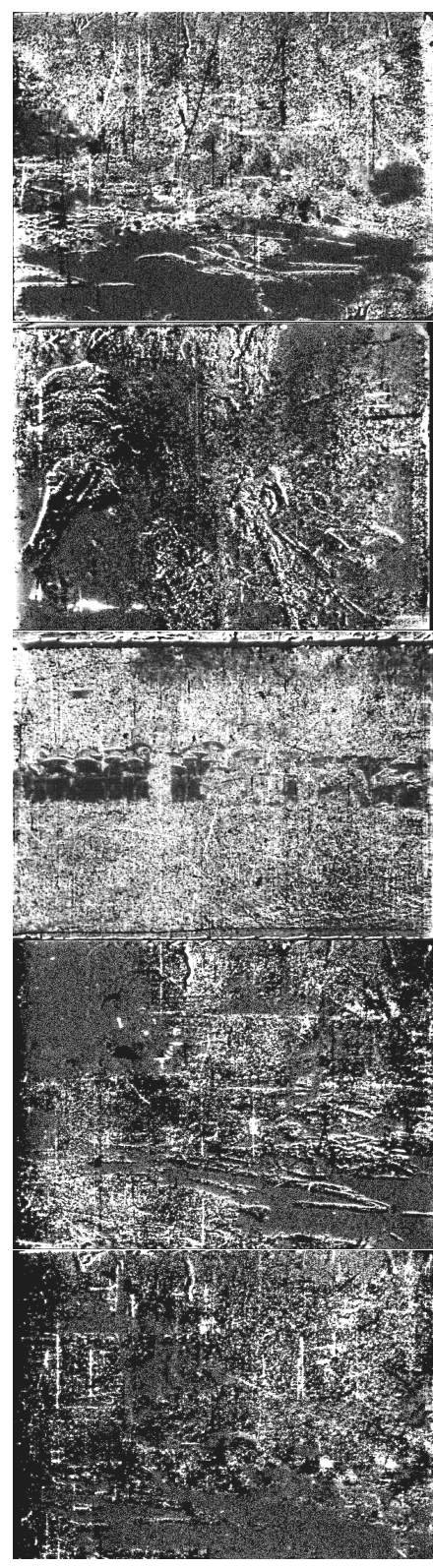

c. OBMA

图 9 不同场景下前后帧差值图像 
BMA 运动补偿后与前一帧参考帧的差值图像; 图 9c 所示为加人 OBMA 运动补偿后与前一帧参考帧 的差值图像.

通过图 9 中 5 个不同场景下运动补偿后的差值 图像对比分析发现，本文提出的运动估计算法的 实际补偿效果与传统块匹配法基本一致.

\section{3 客观评价}

客观评价使用 $1080 \mathrm{P}$ 和 720P 测试视频中的连 续 50 帧视频图像序列作为测试样本. 对比实验分 别为相同清晰度测试视频图像序列、不同匹配块比 例; 相同匹配块、不同清晰度测试视频图像序列.

为了验证匹配块大小对算法性能的影响，测
试实验选择 $1080 \mathrm{P}$ 测试视频图像序列, 2 种不同尺 寸的匹配块作对比实验, 分别是测试图像尺寸的 1/2(540 像素×960 像素)和 1/4(270 像素×480 像素). 图 10a 所示为匹配块尺寸为 1/2(540 像素 $\times 960$ 像 素)时, OBMA 与 BMA 的 PSNR 和运行时间对比图; 图 10b 所示为匹配块尺寸为 1/4(270 像素 $\times 480$ 像 素)时, OBMA 与 BMA 的 PSNR 和运行时间对比图. 为了验证视频清晰度对算法性能的影响, 本 文选择 720P 测试视频与上述 $1080 \mathrm{P}$ 测试视频进行 对比实验. 图 10c 是匹配块尺寸为 1/4(180 像素 $\times$ 320 像素)时, OBMA 与 BMA 的 PSNR 和运行时间 对比图.
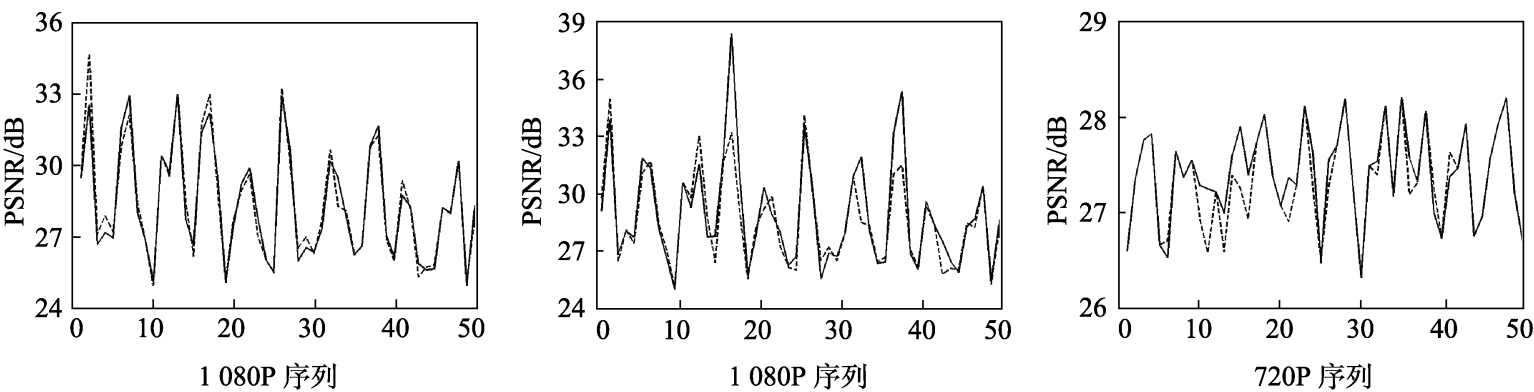

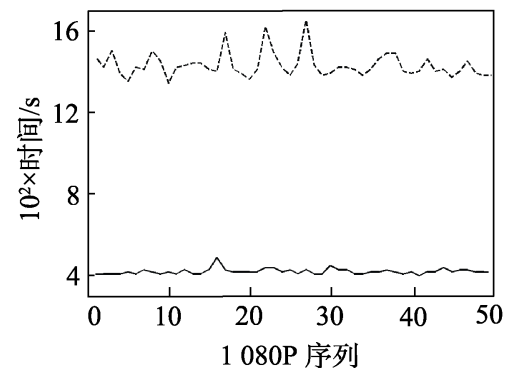

a. 1/2(540像素×960像素)

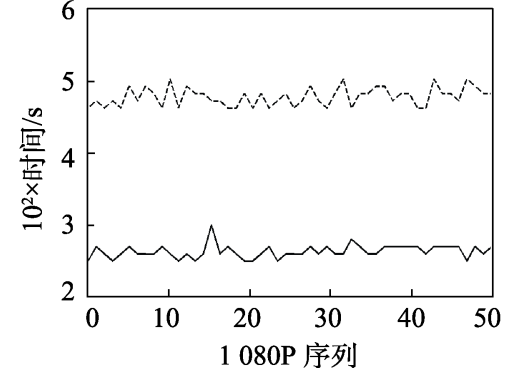

b. $1 / 4(270$ 像素 $\times 480$ 像素 $)$

--- $\mathrm{BMA}^{[3]} ;$ - OBMA

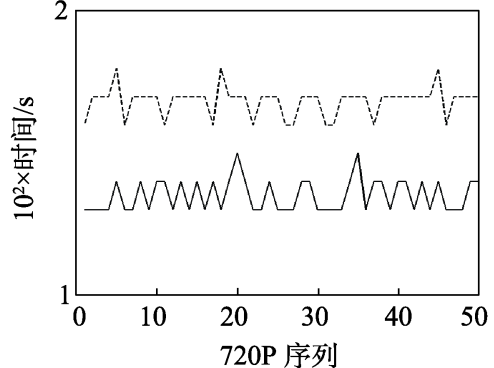

c. $1 / 4(180$ 像素×320像素)

图 10 BMA 和 OBMA 的 PSNR 和运行时间对比

对表 1 中的实验结果进行分析：当匹配块尺寸 和测试视频清晰度相同时, OBMA 与 BMA 的 PSNR 基本相同, 这说明它们具有同样搜索质量. 匹配块为测试图像尺寸的 1/2(540 像素 $\times 960$ 像素), OBMA 的平均运行时间是 BMA 的 29.5\%, 搜索速 度提高 238.6\%; 匹配块为测试图像尺寸的 1/4(270 像素 $\times 480$ 像素), OBMA 的平均运行时间是 BMA 的 59.6\%，搜索速度提高 67.64\%; 匹配块为测试图 像尺寸的 1/4(180 像素 $\times 320$ 像素), OBMA 的平均运 行时间是 BMA 的 $80.1 \%$, 搜索速度提高 $24.72 \%$.

由上述数据分析可知, 与传统的 BMA 相比, 在搜索质量相同的情况下, OBMA 实时性优于 BMA. 运动估计使用的匹配块尺寸越大，搜索速 度提高越明显; 视频清晰度越高, 搜索速度提高越
表 12 种算法连续 5 帧视频图像序列关键指标横向对比

\begin{tabular}{rrrcc}
\hline 算法 & 分辨率 & 匹配块 & $\begin{array}{c}\text { 平均 } \\
\mathrm{PSNR} / \mathrm{dB}\end{array}$ & $\begin{array}{c}\text { 平均搜索 } \\
\text { 时间/s }\end{array}$ \\
\hline \multirow{3}{*}{$\mathrm{BMA}^{[3]}$} & $1080 \mathrm{P}$ & $1 / 2$ & 28.87 & 0.14305 \\
& $1080 \mathrm{P}$ & $1 / 4$ & 28.84 & 0.05259 \\
& $720 \mathrm{P}$ & $1 / 4$ & 27.36 & 0.01685 \\
\hline \multirow{2}{*}{$\mathrm{OBMA}$} & $1080 \mathrm{P}$ & $1 / 2$ & 28.83 & 0.04224 \\
& $1080 \mathrm{P}$ & $1 / 4$ & 29.16 & 0.03137 \\
& $720 \mathrm{P}$ & $1 / 4$ & 27.42 & 0.01351 \\
\hline
\end{tabular}

明显. 本文提出 OBMA 更适用于清晰度较高的视 频. 随着视频分辨率的不断提高, $2 \mathrm{~K}, 4 \mathrm{~K}$ 和 $8 \mathrm{~K}$ 视 频的普及, 运动估计需要的块尺寸也会随之增大, 传统 BMA 庞大的数据量会占据更多的资源, 而使 用本文提出 OBMA 可以有效地解决此问题. 


\section{4 结 语}

目前主流运动估计算法依然停留在直接使用 二维视频图像的二维信息直接计算运动矢量, 本 文通过对视频帧间相关性的研究发现，经过特定 的权重系数矩阵对二维匹配块降维后，一维特征 矩阵具备二维矩阵的部分特征, 使用一维特征矩 阵代替二维矩阵进行运动估计, 减少计算量. 通过 对比实验表明, 本文提出的 OBMA 与 BMA 相比, 在搜索质量相当的前提下，能有效地提高运动估 计的计算速度，具有一定实用价值.

\section{参考文献(References):}

[1] Yu Yinghuai, Wang Jinrong. High accuracy sub-pixel global motion estimation based on upsampled gradient cross-correlation algorithm[J]. Journal of Image and Graphics, 2012, 17(12): 1492-1499(in Chinese)

(余应淮，王锦荣. 高精度亚像素全局运动估计的上采样梯 度互相关算法 [J]. 中国图象图形学报，2012，17(12): 1492-1499)

[2] Li Ziyin, Zhu Shanan. A fast efficient partial distortion search algorithm for block motion estimation[J]. Journal of Image and Graphics, 2006, 11(4): 480-485(in Chinese) (李子印, 朱善安. 一种快速高效的部分失真块运动估计搜 索算法[J]. 中国图象图形学报, 2006, 11(4): 480-485)

[3] Zhao N N, O’Connor D, Basarab A, et al. Motion compensated dynamic MRI reconstruction with local affine optical flow estimation[J]. IEEE Transactions on Biomedical Engineering, 2019, 66(11): 3050-3059

[4] Mukaddim R A, Meshram N H, Mitchell C C, et al. Hierarchical motion estimation with Bayesian regularization in cardiac elastography: simulation and in-vivo validation[J]. IEEE Transactions on Ultrasonics, Ferroelectrics, and Frequency Control, 2019, 66(11): 1708-1722

[5] Qin Rong, Ma Zhiqiang, Zhang Xiaoyan, et al. A fast and robust global motion estimation algorithm[J]. Journal of Air Force Engineering University: Natural Science Edition, 2012, 13(6): 55-59(in Chinese)

(秦荣，马志强, 张晓燕, 等. 一种快速鲁棒的全局运动估计
算法 [J]. 空军工程大学学报: 自然科学版, 2012, 13(6): 55-59)

[6] Tang Jialin, Zheng Jiefeng, Li Xiying, et al. Video stabilization algorithm based on feature matching and motion compensation[J]. Application Research of Computers, 2018, 35(2): 608610(in Chinese)

(唐佳林, 郑杰锋, 李熙莹, 等. 基于特征匹配与运动补偿的 视频稳像算法 [J]. 计算机应用研究, 2018, 35(2): 608-610)

[7] Liu Haihua, Lei Yi, Xie Changsheng. Fast block-matching motion estimation based on a dual-cross search algorithm[J]. Computer Research and Development, 2006, 43(9): 1666-1673(in Chinese)

(刘海华, 雷奕, 谢长生. 双十字搜索算法的快速块匹配运 动估计[J]. 计算机研究与发展, 2006, 43(9): 1666-1673)

[8] Li Hejun, Li Heping, Li Jianxiong. A multi-pattern switching algorithm for fast motion estimation[J]. Journal of Electronics \& Information Technology, 2013, 35(3): 689-695(in Chinese) (李贺军, 李和平, 李建雄. 一种采用多模式切换的快速运 动估计算法[J]. 电子与信息学报, 2013, 35(3): 689-695)

[9] Li Qiaoliang, Wang Guoyou, Zhang Guilin, et al. Accurate global motion estimation based on pyramid with mask[J]. Journal of Computer Aided Design \& Computer Graphics, 2009, 21(6): 758-762(in Chinese)

(李乔亮, 汪国有, 张桂林, 等. 基于掩模金字塔的高精度全 局运动估计算法 $[\mathrm{J}]$. 计算机辅助设计与图形学学报, 2009, 21(6): 758-762)

[10] Zhang Maolei, Chen Jianguo, Yuan Hongyong, et al. Video stabilization on a six-rotor aircraft platform[J]. Journal of Tsinghua University: Science and Technology, 2014, 54(11): 1412-1416(in Chinese)

(张毛否，陈建国，袁宏永，等. 六旋翼飞行平台的视频稳像 技术[J]. 清华大学学报: 自然科学版, 2014, 54(11): 1412-1416)

[11] Zhang T, Fei S M, Li X D, et al. Fast global motion estimation and moving object extraction algorithm in image sequences[J]. Journal of Southeast University: English Edition, 2008, 24(2): 192-196

[12] Li R X, Zeng B, Liou M L. A new three-step search algorithm for block motion estimation[J]. IEEE Transactions on Circuits and Systems for Video Technology, 1994, 4(4): 438-442

[13] Xu Jin. Research on key technology of digital restoration of motion picture film[D]. Shanghai: Shanghai Jiaotong University, 2009(in Chinese)

(徐进. 电影胶片数字修复关键技术研究 $[D]$. 上海: 上海交 通大学, 2009) 